\title{
Effets d'une consommation prolongée de "koutoukou» (boisson alcoolique artisanale) sur la mémoire spatiale chez la souris.
}

\author{
BADJO Pierrette ${ }^{1}$, DIBOH Emmanuel ${ }^{2}$, GBALOU Kakou Luc ${ }^{1}$, ADOU K. Fiéni. Jean-Baptiste ${ }^{1}$, \\ ZUNON-KIPRE Ivan ${ }^{3,4}$, TAKO Némé Antoine1. \\ 1 : Laboratoire de Neurosciences - UFR Biosciences - Université de Félix Houphouët Boigny de Cocody. \\ 2 : Université Jean Lorougnon Guédé (DALOA) \\ 3 : Laboratoire d'anatomie, UFR SMA- Université de Félix Houphouët Boigny de Cocody. \\ 4 : Service de Neurochirurgie, CHU de Yopougon-Abidjan. \\ Correspondance email : emmanueldiboh@gmail.com \\ Adresse : BP 150 DALOA / Téléphone : +225-07 1978 93 / + 225-04 332249
}

Original submitted in on $4^{\text {th }}$ July 2017. Published online at www.m.elewa.org on $31^{\text {st }}$ August 2017 http://dx.doi.org/10.4314/jab.v116i1.4

\section{RESUME}

Objectif : le but de cette étude était de déterminer les effets du «koutoukou », une boisson traditionnelle issue de la distillation de sève fermentée de palmier à huile (Elaeis guineensis Jacq) sur les aptitudes mnésiques.

Méthodologie et Résultats : 62 souris de souche swiss dont 22 témoins et 40 animaux expérimentaux ont été soumis à une épreuve du labyrinthe en $\mathrm{T}$. Les témoins consommaient de l'eau ordinaire comme boisson. Les animaux expérimentaux ont été répartis en deux groupes : 20 souris alcoolisés au koutoukou durant 3 mois et 20 autres souris alcoolisées au koutoukou durant 6 mois. La comparaison du taux d'alternance spontanée sérielle entre les deux groupes expérimentaux a montré une diminution plus marquée de ce taux (alternance) chez les animaux alcoolisés durant 6 mois. Quant aux épreuves d'alternance différées, elles ont montré que ce taux chute significativement dès 6 heures (délai entre la phase d'acquisition et la phase de restitution) chez les souris traitées au koutoukou durant 6 mois contre 24 $\mathrm{H}$ chez ceux alcoolisé pendant 3 mois.

Conclusion et application des résultats : la consommation chronique de koutoukou entraine des déficits tant de la mémoire de travail que de la mémoire épisodique.

Mots clés : alcoolisation, koutoukou, mémoire, souris.

\section{ABSTRACT}

Objective: The aim of this study was to determine the effects of "koutoukou", a traditional drink after the fermented sap of distilling oil palm (Elaeis guineensis) on memory skills

Methodology and Results: 62 Swiss mice of which 22 controls and 40 experimental animals were subjected to a T-labyrinth test. The controls consumed ordinary water as a drink. The experimental animals were divided into two groups: 20 koutoukou alcoholic mice for 3 months and 20 other koutoukou alcoholic mice 
for 6 months. Comparison of the serial spontaneous alternation between the two experimental groups showed a more marked decrease in this rate (alternation) in the alcoholic animals during 6 months. As for the delayed alternation of tests, they showed that this rate drops significantly at 6 am (the time between the acquisition phase and the return phase) in mice treated koutoukou for 6 months against 24 hours in those alcohol for 3 month.

Conclusion and application of results: Chronic koutoukou consumption leads to deficits in both working memory and episodic memory.

Keywords: Alcohol, koutoukou, memory, mouse.

\section{INTRODUCTION}

L'alcool altère la plupart des fonctions cérébrales, ce qui réduit temporairement les aptitudes mnésiques, l'aptitude à réfléchir, à voir et à agir (Weiss et al., 2014; Harnett et al., 2013). En Afrique sub-saharienne, en 2010, la consommation d'alcool a été le principal facteur de risque de décès et d'invalidité (Lim et al., 2012). Une enquête effectuée par l'OMS en 2005, a révélé que $71 \%$ de la population ivoirienne consommerait de l'alcool au moins une fois dans l'année. Parmi cette population, $77,9 \%$ des hommes et $22,1 \%$ des femmes ingèrent plus de 5 verres de boissons alcooliques chaque fois qu'ils ont l'occasion de boire (Anonyme, 2016). En outre, une étude épidémiologique réalisée sur 3428 sujets des deux sexes, montre que les boissons locales, dont fait partie le Koutoukou représentent l'essentiel de la consommation alcoolique en Côte d'Ivoire, surtout en région rural (Yao et al., 2012). Cette enquête indique que la consommation régulière est plus importante chez les hommes $(74,8 \%)$ que chez les femmes $(61,8 \%)$. Ce phénomène touche toutes les couches de la

\section{MATERIEL ET METHODES}

Matériel biologique: Notre matériel biologique se composait de souris mâles de souche swiss provenant de l'Institut Pasteur. Les mâles ont été préférés aux femelles; plus sujettes aux influences hormonales. Ces animaux ont été fournis par lots homogènes de 15 souris, âgées de 4 à 5 semaines $(14-17 \mathrm{~g})$. À leur arrivée au laboratoire, elles ont été placées en cages collectives. À l'âge de 9 semaines, les souris ont été soumises aux différents traitements avant utilisation. Notre échantillon se composait de 62 souris réparties comme suit :

$\mathrm{ST}(\mathrm{n}=22$ ): souris témoins (eau ordinaire comme boisson) ; population ivoirienne à tel enseigne qu'un autre sondage réalisé par Diboh (2013), mentionne que parmi 2854 élèves (10 à 24 ans) interrogés, 79,47 $\%$ affirment avoir consommé au moins une fois une boisson alcoolique tandis que $18 \%$ en consomment régulièrement. Aussi, vu sa disponibilité, le Koutoukou artisanal est autant consommé $(9,39 \%)$ que préféré $(9,93 \%)$. Cependant, cette boisson, issue de la distillation de la sève fermentée de palmier à huile (Elaeis guineensis Jacq.), semble plus nocive que les autres boissons alcooliques dites industrielles. En effet, il a été prouvé que la prise aiguë de ce breuvage a des effets très prononcés sur la vigilance (Camara, 2002; Diboh, 2006 et 2015, Yao, 2009). Aussi, régulièrement consommée, cette substance serait potentiellement épileptogène (Diboh, 2014). Ainsi, l'objectif de cette étude est de déterminer les effets du koutoukou de fabrication artisanale sur les aptitudes mnésiques, en se servant d'un modèle animal.

SK3 $(n=20)$ : souris alcoolisées au Koutoukou durant 3 mois ;

SK6 $(n=20)$ : souris alcoolisées au Koutoukou durant 6 mois.

Matériel technique: Ce dernier est essentiellement composé d'un alcoomètre centésimal de type GAY LUSSAC, de cages d'élevage, de biberons, de boissons alcooliques (éthanol pur et Koutoukou) et d'un labyrinthe en $T$ (figure 1). Le labyrinthe en $T$ est construit en plexiglas de couleur grise. II comprend un compartiment de départ (15 cm de long), un couloir central (45 cm de long) et deux branches d'arrivée identiques de $31 \mathrm{~cm}$. Tous les compartiments ont la 


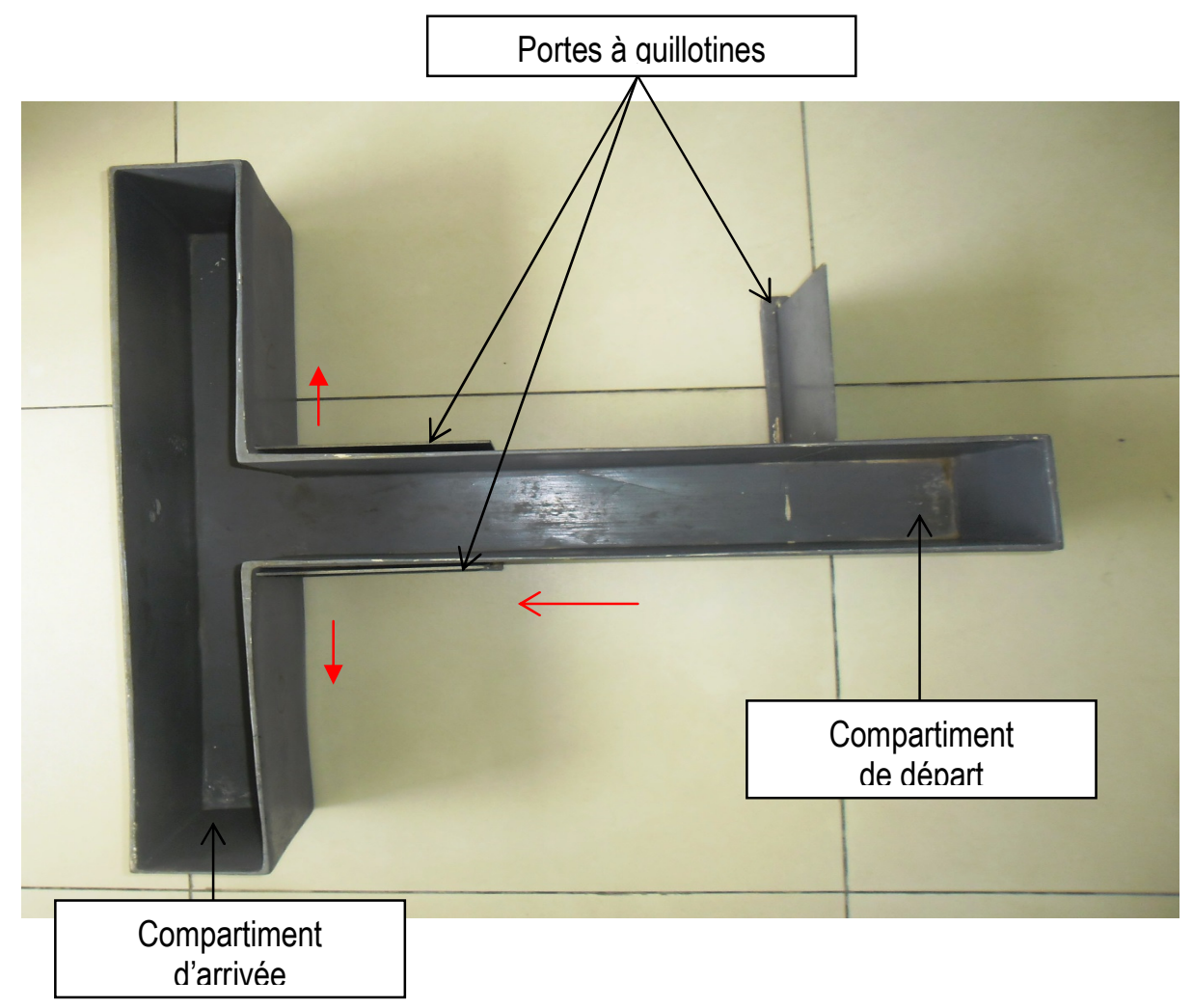

Figure 1 : labyrinthe et $\mathrm{T}$

La souris, une fois placée dans le compartiment de départ peut se diriger dans chacune des deux branches d'arrivée. Toute fois son trajet peut lui être imposé selon le cas de figure, en fermant l'une des deux portes à guillotine des compartiments d'arrivée.

\section{METHODES}

Au plan méthodologique, l'étude a comporté plusieurs étapes.

- Solution alcoolique : Le koutoukou pur fortement titré (46\%), a été dilué à des concentrations de $23 \%$, $12 \%$ et $8 \%$ pour les séances d'alcoolisation. Ainsi, après avoir procédé à l'évaluation du titre alcoolique de la solution mère (boisson) au moyen de l'alcoomètre centésimal de GAY LUSSAC qui a indiqué $46^{\circ}$, la préparation de la solution alcoolique s'est faite par dilution en vue d'obtenir les concentrations recherchées. Cette dilution est opérée en suivant la formule

$\mathrm{CiVi}=\mathrm{CfVf}$ où :

Ci représente la concentration initiale ;

Cf est la concentration finale ;

Vi correspond au volume initial ;et

Vf indique le volume final.
Ainsi, à partir de $500 \mathrm{ml}$ d'une solution-mère de Koutoukou à $46^{\circ}$, contenue dans une éprouvette graduée, l'obtention d'une solution diluée à $23 \%$ s'est faite par ajout de $500 \mathrm{ml}$ d'eau distillée.

- Alimentation et alcoolisation : Les animaux ont reçu ces traitements en cage collective par groupe de 5. Ils ont été nourris ad libitum avec des granulés et un mélange de poissons séchés, d'arachides et de pain sec (Yao et al., 2010). Les biberons contenant les boissons étaient accrochés au-dessus des cages. Ces animaux ont été maintenus dans des conditions standard de nourriture, de température et d'éclairage. Toutes ces souris ont eu le même régime alimentaire, seules différaient les boissons. L'administration des solutions alcooliques s'est fait de manière progressive : solution à $8 \%$ la première semaine, $12 \%$ la seconde et $23 \%$ la troisième semaine. Les mesures de consommations dipsique et alimentaire ont été 

alcoolique artisanale) sur la mémoire spatiale chez la souris.

effectuées deux fois par semaine. Après la période de traitement ( 3,6 ou 12 mois), le sevrage s'est fait également de manière dégressive : $12 \%$ la première semaine, $8 \%$ la seconde pour ne recevoir que de l'eau dans la troisième semaine et ceci durant au moins 3 semaines. Les souris ont été ensuite isolées en cages individuelles.

Phase d'habituation: Les animaux ont été soumis à des séances d'exploration libre (5 à 10 min par jour pendant 3 jours). Ces séances étaient destinées à atténuer leurs réactions émotionnelles au contact de l'expérimentateur (qui les manipule quotidiennement en les pesants à la même heure de la journée) et du contexte (labyrinthe en $\mathrm{T}$ et environnement expérimental).

Phase expérimentale: Les animaux ont subi deux types d'épreuve à savoir l'alternance spontanée sérielle et l'alternance différée, chacune d'elles mesurant un aspect distinct de la mémoire.

- Alternances spontanées sérielles: Les souris ont été soumises à une séance de 6 essais successifs dits "libres" car à chaque essai l'animal pouvait choisir d'aller dans l'une ou l'autre branche d'arrivée. Chaque essai s'est déroulé de la façon suivante : l'animal était enfermé pendant 30 secondes (s) dans le compartiment de départ. Au terme de ce délai, la porte à guillotine est ouverte et la souris pouvait choisir l'une des deux branches où elle a été enfermée pendant 30 $\mathrm{s}$. Après ce délai, elle était, à nouveau placée dans la boite de départ pour 30 autres secondes et ainsi de suite. L'intervalle séparant chaque essai ou intervalle entre essai (I.E.E) a été de 30 secondes.

- Alternance différée : Afin d'étudier le décrément du taux d'alternance entre deux essais pour des intervalles pouvant aller jusqu'à 48h (et même, dans certains cas, à $72 \mathrm{~h}$ ), la procédure dite des "essais forcés" a été utilisée. Dans ce cas de figure, l'animal a été forcé d'entrer deux fois de suite dans la même branche (I.E.E : $30 \mathrm{~s}$ ) (acquisition), l'accès à l'autre branche étant interdit par la fermeture de la porte. Après un délai variable $(5 \mathrm{~min}, 6 \mathrm{~h}, 24 \mathrm{~h}, 48 \mathrm{~h}$ et même 72 heures), l'animal fut placé à nouveau dans le labyrinthe pour un essai libre de rétention, l'accès aux deux compartiments d'arrivée étant libre. Pour chaque

\section{RÉSULTATS}

\section{Alternances spontanées sérielles}

A 3 mois d'alcoolisation: Dans l'ensemble, les performances des animaux alcoolisés durant 3 mois n'étaient pas significativement différents de celles de témoins $[F(1,26)=3,95 ; p=0,083]$. La comparaison épreuve d'alternance différée (comportant 2 essais forcés à droite par exemple +1 essai test), une seconde épreuve a été effectuée où les deux essais forcés d'acquisition étaient réalisés à gauche (dans notre exemple) afin d'éviter d'éventuelles distorsions des résultats dues aux préférences (ou latéralisation). La deuxième précaution a consisté, après chaque épreuve d'alternance différée, à effectuer une séance d'exploration libre $(5 \mathrm{~min})$ de façon à provoquer une sorte de remise à zéro (cette procédure permet de rééquilibrer l'attirance relative que l'animal doit avoir pour les deux bras).

Analyse statistique: Le caractère étudié a été le nombre d'alternance par série d'essai, traduit en pourcentage. Ainsi, le pourcentage d'alternance a été déterminé de la manière suivante :

Nombre d'alternance pour la série d'essai considéré $\times 100$ Nombre maximal d'alternance

Par ailleurs, à chaque série d'expériences, trois groupes d'animaux étaient à comparer : les animaux témoins, les animaux tests au Koutoukou de vin de palme (après 3 mois d'alcoolisation) et les animaux tests au Koutoukou de vin de palme (après 6 mois d'alcoolisation). Le comportement d'ensemble de chaque groupe a été analysé à travers leurs performances. Ces résultats ont été confrontés aux autres groupes. Par ailleurs, il a été procédé à la vérification de la significativité des différences probables observées entre les moyennes des alternances obtenues dans chaque groupe. C'est-à-dire savoir si pour chaque épreuve la différence de performances entre deux groupes donnés était significative ou pas. Pour ce faire, une analyse de variance (ANOVA) univariée, grâce au logiciel Statistica 10.0, a permis de faire ces comparaisons. La probabilité $(p)$ de 0,05 est considérée comme valeur limite de significativité. Ainsi, si « $p$ » est inférieur ou égale à 0,05 , alors la différence entre les variables comparées est significative. Par contre, si « $p$ » est supérieur à 0,05 , la différence entre les deux variables comparées n'est pas significative.

intragroupe des performances a montré que le taux d'alternance sérielle ne varie quasiment pas chez les témoins quel que soit le nombre d'essai, à l'exception de l'essai 3 pour lequel il est observé une régression de $6,41 \%(p \leq 0,048)$. En revanche, chez les animaux 

alcoolique artisanale) sur la mémoire spatiale chez la souris.

alcoolisés, ce pourcentage a baissé au fur et à mesure que le nombre d'essai augmente (figure 1A). Ainsi, des différences significatives dans l'évolution des alternances ont été observées au niveau des essais 4 , 5 et 6 . II s'agit respectivement de :[F $(1,43)=17,52 ; p$ $=0,0025],[F(1,43)=31,637 ; p=0,001]$ et $[F(1,43)=$ $9,57 ; p=0,003]$.

A 6mois d'alcoolisation: Dans l'ensemble, les performances des animaux alcoolisés durant 6 mois ont différé très significativement de celles des témoins $[F$
$(1,26)=9,995 ; p=0,003]$. Par ailleurs, tout comme à 3 mois d'alcoolisation, les performances des témoins ne varient quasiment pas. Par contre, comme le montre la figure $1 \mathrm{~B}$, chez les animaux expérimentaux, l'augmentation du nombre d'essais a entrainé une diminution significative et progressive du taux d'alternance sérielle dès l'essai $3(p \leq 0,001)$. On note aussi qu'à partir du $4^{\mathrm{e}}$ essai, les souris ont répondu au hasard.
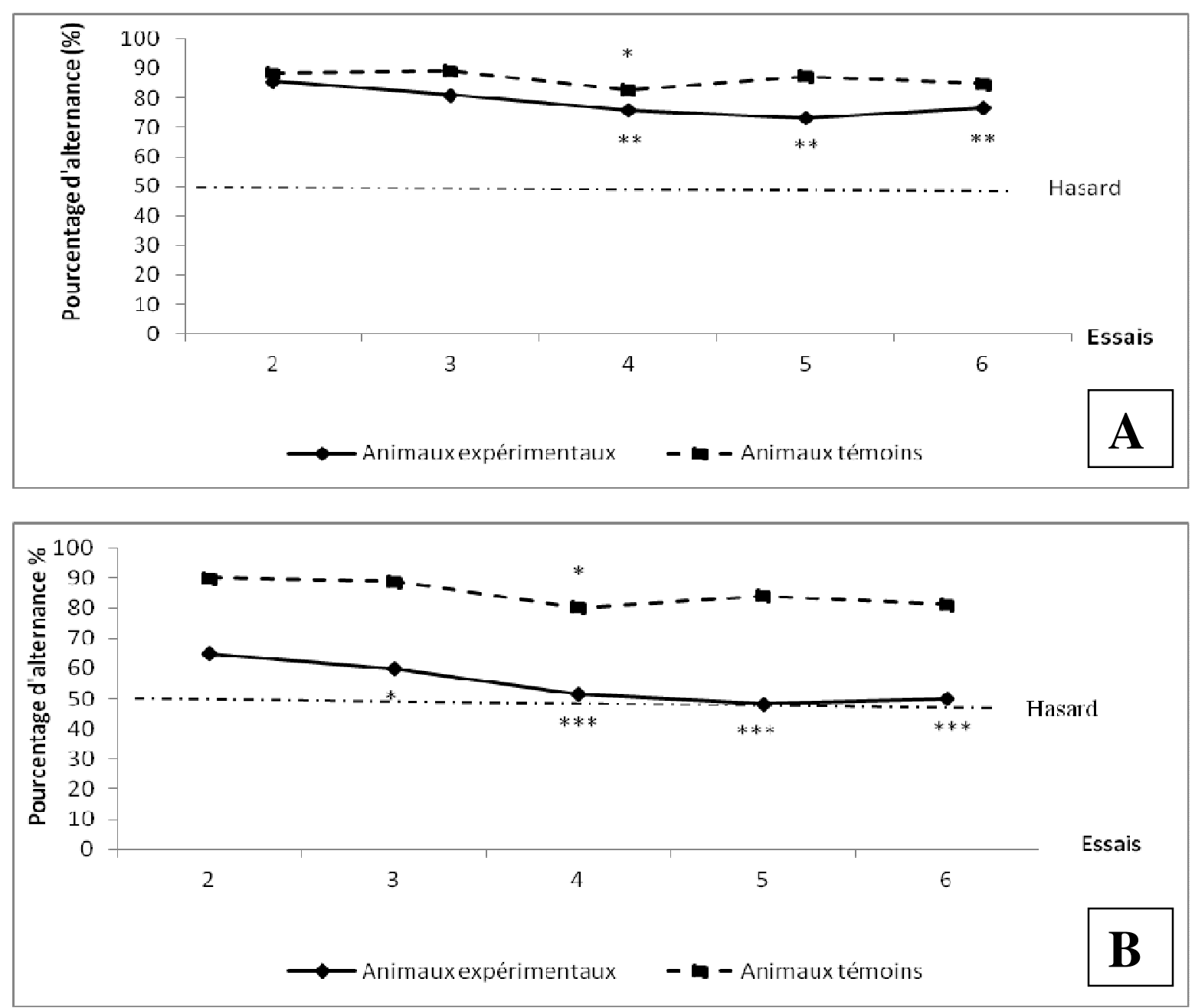

Figure 2: évolution des taux d'alternances spontanées sérielles des témoins et des animaux alcoolisés au koutoukou durant 3 mois $(A)$ et durant 6 mois $(B)$.

En $A$, une baisse significative et croissante des performances est observée en fonction du nombre d'essai chez les animaux expérimentaux.

En $B$, dès le $4^{e}$ essai, les animaux alcoolisés au Koutoukou durant 6 mois répondent au hasard.

Les facteurs de significativité indiqués impliquent uniquement la comparaison intragroupe (comparaison des performances en fonction des essais soit chez les témoins ou soit chez les animaux expérimentaux).

${ }^{*}: p \leq 0,05 ;{ }^{* *}: p \leq 0,01 ;{ }^{* *}: p \leq 0,001$ 

alcoolique artisanale) sur la mémoire spatiale chez la souris.

A 12 mois d'alcoolisation: A 12 mois d'alcoolisation, les échantillons disponibles n'étaient pas statistiquement significatifs à cause du fort taux de mortalité des animaux $(80 \%$ de perte après 8 mois d'alcoolisation au koutoukou). Une comparaison effectuée entre les animaux traités au koutoukou durant 3 mois et ceux de 6 mois, a montré une diminution très significative du taux d'alternance chez les animaux alcoolisés pendant 6 mois $[F(1,36)=7,9987 ; p=$ 0,007].

\section{Alternances différées}

A 3 mois d'alcoolisation: La comparaison des performances entre les animaux expérimentaux et témoins révèle que pour les intervalles de rétention relativement courts (figure $2 \mathrm{~A}$ ), notamment à 5 minutes et $6 \mathrm{~h}$, il n'y a pas de différence significative [ $5 \mathrm{~min}: \mathrm{F}$ $(1,35)=1,057$ pour $p=0,310)$ et $6 h: F(1,24)=$ $0,736$ pour $p=0,396]$. Cependant, à $24 \mathrm{~h}$ les performances des souris traitées au Koutoukou artisanal diffèrent très significativement des témoins $[F$ $(1,34)=9,714 ; p=0,0037]$.

- A 6 mois d'alcoolisation: Pour l'intervalle $5 \mathrm{mn}$, les animaux alcoolisés pendant 6 mois et les témoins ont des performances quasiment similaires $(87,17 \%$ vs $88,24 \%$ ). II n'y a donc pas de différence significative car $F(1,23)=0,26101$ et $p=0,61430)$. L'augmentation de l'intervalle à $6 \mathrm{~h}$ entraine une réduction significative $\mathrm{du}$ taux d'alternance des animaux alcools uniquement $(61,21 \%$ vs $72,22 \% ; p=0,039)$. Comme le montre la figure $2 \mathrm{~B}$, cette baisse du taux d'alternance se poursuit aux intervalles $24 h, 48 h$ et $72 h(p=0,0410 ; p=0,003 ; p$ $=0,0265)$. La comparaison entre les 2 groupes expérimentaux (alcool 3 mois et alcool 6 mois) a montré qu'à 5 minutes d'intervalle de rétention, tous les animaux ont un bon niveau de rétention (alcool 3 mois : $88,65 \%$ vs $87,17 \%$ : alcool 6 mois). Cependant, le taux d'alternance est significativement différent pour l'intervalle de 24h $[F(1,29)=5,739 ; p=0,02327]$. Audelà de ce délai, ces animaux répondent au hasard.

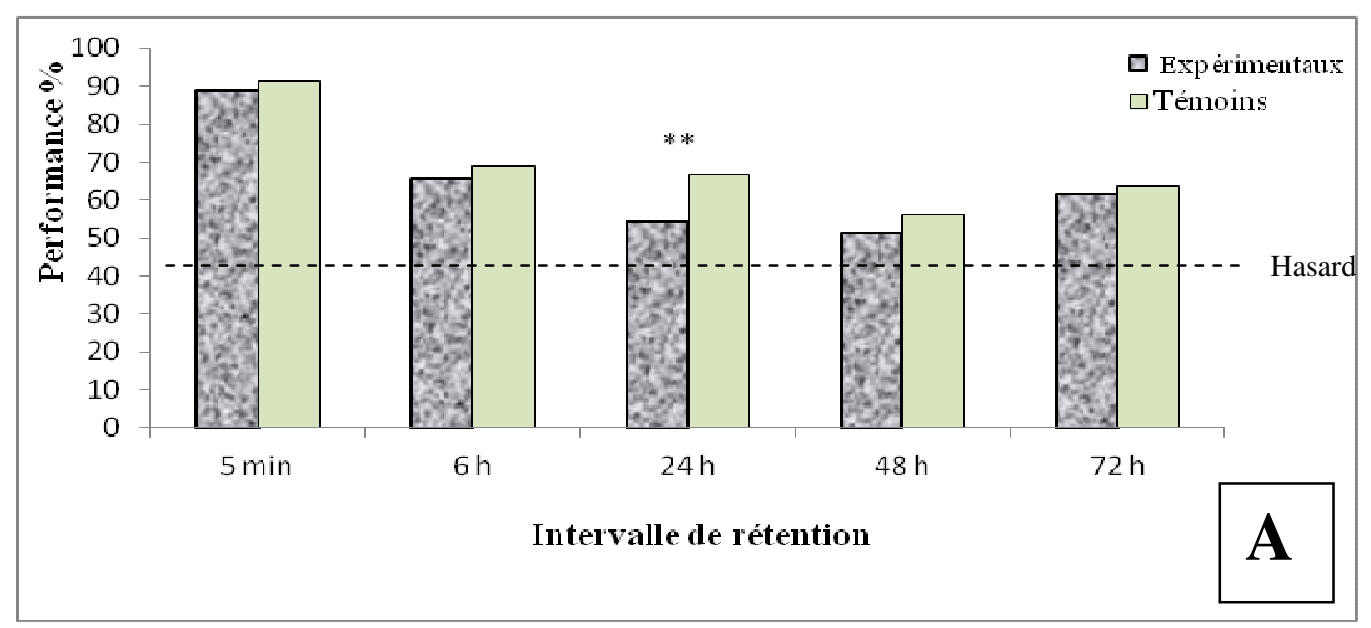



alcoolique artisanale) sur la mémoire spatiale chez la souris.

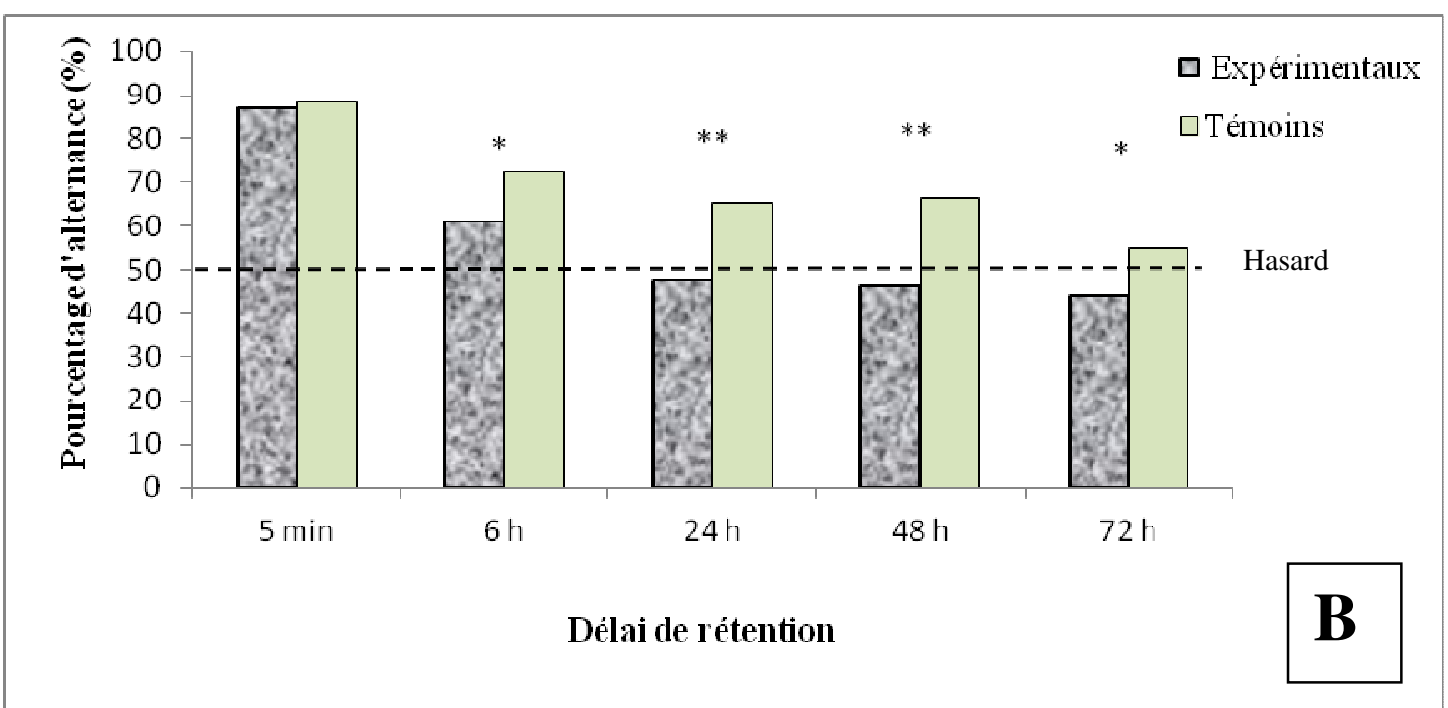

Figure 3 : évolution du taux d'alternance en fonction des intervalles de rétention (5 min, $6 \mathrm{~h}, 24 \mathrm{~h}, 48 \mathrm{~h}$ et $72 \mathrm{~h}$ ), chez les souris traitées au koutoukou pendant 3 mois (A) et 6 mois (B) et leurs témoins respectifs.

En $A$, on note une baisse significative du taux d'alternance des animaux expérimentaux pour lintervalle de $24 \mathrm{~h}$.

En $B$, les performances des animaux traités diminuent significativement et de manière progressive en fonction du délai de rétention et ceci dès $6 \mathrm{~h}$ d'intervalle.

Les facteurs de significativité indiqués impliquent uniquement la comparaison intergroupe (entre animaux témoins et animaux expérimentaux).

${ }^{*}: p \leq 0,05 ;{ }^{* *}: p \leq 0,01 ;{ }^{* * *}: p \leq 0,001$

\section{DISCUSSION}

L'alternance est une épreuve qui permet de mettre en évidence la capacité de la souris à alterner, et éventuellement la détérioration progressive de son comportement au fur et à mesure que le nombre d'essais augmente. En effet, on peut supposer que la réponse à l'essai $n$ dépendant de la mémoire du bras visité à l'essai $(n-1)$ peut être perturbée par les souvenirs des choix antérieurs (de 1 à n-2). L'épreuve permet donc d'étudier la sensibilité aux interférences proactives (Beracochea et al.,1987), comme observé chez les témoins à l'essai 3 . En outre, les résultats ont révélé qu'après 3 mois de consommation de koutoukou, au cours des épreuves d'alternances sérielles spontanées, l'augmentation du nombre d'essais entraine une diminution significative du taux d'alternances chez les animaux soumis à l'alcoolisation chronique. La comparaison du taux global des animaux alcools 3 mois et ceux de 6 mois montre que la diminution du taux d'alternances est progressive. $\mathrm{Ce}$ résultat montre une sensibilité exagérée aux interférences et aussi un déficit de la mémoire de travail de ces animaux. De même, les épreuves d'alternances différées entrainent chez les alcools 3 mois une chute du taux d'alternance sur l'intervalle de rétention long, notamment à partir de $24 \mathrm{~h}$. Ce déficit est aussi observé sur l'intervalle court de $6 \mathrm{~h}$ après 6 mois d'alcoolisation. Cette baisse de performance met en évidence un déficit de la mémoire à long terme (mémoire épisodique) chez les animaux traités au Koutoukou, comme mentionné par Tako et al., (1988).Une autre étude réalisée par Yao et al., (2011) sur des sujets humains adultes indique que les effets d'une perturbation mnésique engendrée par le Koutoukou artisanal durent 3 à $4 \mathrm{~h}$ plus longtemps que celle produite par des boissons industrielles notamment le Pastis 45. Dans le même ordre d'idée, Diboh (2014) montre que cette substance entraine une perturbation de la vigilance, de l'attention, mais aussi et surtout de la mémoire de travail chez les élèves consommateurs réguliers de ce breuvage traditionnel. Ces observations confirment l'hypothèse selon laquelle le Koutoukou de vin de palme contiendrait d'autres molécules capables de potentialiser les effets de l'éthanol (Hamon et al., 2002) par compétition sur l'alcool déshydrogénase, lors du métabolisme. Ainsi, le méthanol, le butanol, le propanol et les autres éléments chimiques contenus dans le Koutoukou de vin de palme pourraient être à la base des lésions du cortex cérébral observé par Yao et al., (2011) sur les cerveaux de rats (rattus norgevicus) traités au Koutoukou. Ces substances seraient aussi la 

alcoolique artisanale) sur la mémoire spatiale chez la souris.

cause de la baisse significative, dans le temps, des performances des sujets ayant consommé ce breuvage (Camara et al., 2004). A 12 mois d'alcoolisation, le fort

\section{CONCLUSION}

II ressort de cette étude que la consommation chronique de koutoukou artisanal a engendré chez les souris des déficits tant de la mémoire de travail que de la mémoire épisodique. Cependant l'étendu des déficits cognitifs est fonction (proportionnel) de la durée d'alcoolisation. En collaboration avec les autorités

\section{RÉFÉRENCES}

Beracochea D, Lescaudron L, Tako A, Verna A, Jaffard $R, 1987$. Build-up and release from proactive interference during chronic ethanol consumption in mice: a behavioural and neuroanatomical study. Behav. Brain Res., 25: 63-74.

Camara PA, 2002. Alcoolisation au Koutoukou en Côte d'Ivoire : constat et propositions. Alcoologie et Addictologie, 24 (4) : 319-328.

Camara PA, Yao KM, Adou KFJ-B, 2004. Étude préliminaire des effets d'une prise unique de Koutoukou sur le niveau de vigilance et émotionnel de l'Homme. Rev Inter. Sci. de la Vie et de la Terre,4 (1): 99-113.

Anonyme, 2016. Consommation d'alcool. Aidemémoire, $\mathrm{N}^{\circ} 349,678 \mathrm{p}$.

Diboh E, 2006. Analyse épidémiologique de la consommation d'alcool chez les lycéens et les collégiens : effet sur les niveaux de vigilance sous imprégnation au Koutoukou. Mémoire de Diplôme d'Études Approfondies de physiologie animale, cellulaire et membranaire, (option: Neurosciences et Pharmacopée Africaine). Université de Cocody-Abidjan, UFR Biosciences, $50 \mathrm{p}$.

Diboh E, 2013.Alcoolisation chez les jeunes élèves en Côte d'Ivoire : préférence et consommation effective. European Scientific journal, 30 (9): 380-393.

Diboh E, 2014. Effets d'une alcoolisation aigue au koutoukou sur l'attention et la mémoire des jeunes scolarisés de la ville d'Abidjan (Côte d'lvoire). Thèse pour l'obtention du titre de Docteur l'Université Félix Houphouet-Boigny, $146 \mathrm{p}$.

Diboh E, Assi B, Yao K M, Badjo P, Gbalou K L, Tako N A, 2015. Effets du Koutoukou sur L'Electroencéphalogramme (EEG) des jeunes pourcentage de décès observé pourrait s'expliquer par des lésions hépatiques irréversibles, comme mentionné par Yao et al., (2010).

compétentes, la confection de spots publicitaires doit être envisagée, mettant en exergue les effets délétères de cette substance sur les capacités cognitives des consommateurs, appuyant par la même occasion, les actions de la croix bleue ivoirienne.

scolarisées de la ville d'Abidjan (Côte d'Ivoire). Afrique Biomédicale ; 20 : 60-75.

Hamon JF, Camara PA, Adou KFJ-B, Yao KM, 2002. Goûts et habitude en matière de consommation d'alcool dans le sud et le centre-nord de la Côte d'Ivoire : enquête sur 3 428 sujets. Afrique Biomédicale, 7(3) : 19-26.

Harnett PH, Lynch SJ, Gullo MJ, Dawe S, Loxton N, 2013. Personality, cognition and hazardous drinking: Support for the 2-Component Approach to Reinforcing Substances Model. Addict Behav.,38(12):2945-2948.

Lim SS, Vos T, Flaxman AD, 2012. The burden of disease and injury attributable to 67 risk factors and risk factor clusters in 21 regions 1990-2010: A systematic analysis. Lancet, 380, 2224-2260.

Tako AN, Beracochea D J, Jaffard R. 1988. Accelerated rate of forgetting of spatial information following mammillary-body lesions in mice: effects of context change on retention-test performance. Psychobiology, 16, 45-53.

Weiss E, Singewald EM, Ruepp B, Marksteiner J, 2014. Alcoholinduced cognitive deficits. Wien Med Wochenschr, 164 (1-2):9-14.

Yao GV, Dalmeida M-A, Effi AB, Koffi KE, Tre-Yavo M, Doukoure B, Troh E, N'guessan AA, Koffi DK, Kati-Coulibaly S. 2010. Étude microscopique et biochimique des lésions hépatiques du rat adulte albinos, rattusnorgevicus, induite par l'alcoolisation au «koutoukou». Revue africaine de pathologie, 9 (2) : 21-29.

Yao GV, Koffi KE, Doukouré B, D'almeida M-A, TreYavo M, Effi AB, Troh E, N'guessan AA, Koffi KD, Kati-Coulibaly S, 2011. Lésions cérébrales du rat adulte albinos, rattus norgevicus, induite par l'alcoolisation au 
«koutoukou ».Revue africaine de pathologie, $10(1-2): 3-10$.

Yao KM, 2009. Approche épidémiologique de la consommation d'alcool en Côte d'Ivoire et évaluation des effets de l'alcoolisation (aigüe et chronique) au Koutoukou (eau-de-vie de vin de palme) sur le fonctionnement cérébral des consommateurs. Thèse de doctorat en Physiologie Animale, Université de CocodyAbidjan, $N^{\circ} 593,151 p$.

Yao KM, Adou KFJ-B, Camara PA, Bakou NF, Tako NA, Seri B, 2011. Effets comparés de I'alcoolisation aiguë au Koutoukou de vin de palme (boisson alcoolique artisanale) et au Pastis 45 (boisson alcoolique industrielle) sur la mémorisation, chez l'homme. Int. J. Biol. Chem. Sci., 5(3): 1073-1081.

Yao KM, Camara PA, Adou KFJ-B, 2012. Types de boissons alcooliques consommées en Côte d'Ivoire. Préférence et consommation effective. Alcoologie et Addictologie, 34(3): 185-193. 